\title{
GERENCIAMENTO DOS RESIDUOS DE SERVIÇOS DE SAÚDE EM NATAL/RN: RELEITURA DE DIAGNÓSTICOS PRETÉRITOS
}

\section{WASTE MANAGEMENT OF HEALTH SERVICES IN NATAL / RN: DIAGNOSTICS REREADING OF PAST TENSES}

\author{
Ana Ruthinara M. A. Arantes' ${ }^{1}$, Carlos Enrique de M. Jerônimo (Orientador)² \\ 'Especialização em Pericia e Gestão Ambiental. anaruthinaratouros@hotmail.com \\ ${ }^{2}$ Doutorado em Engenharia Quimica. Engenheiro de Processamento de Petroleo da Petrobras. \\ c enrique@hotmail.com
}

http://dx.doi.org/10.5902/223613087652

\begin{abstract}
RESUMO
A problemática com a temática resíduos sólidos é freqüente nas principais pesquisas científicas da área ambiental, entretanto, tal tema sob o ponto de vista legal só teve fortes impulsionamentos com a publicação de resoluções do CONAMA, já datadas no século XXI e pela publicação recente da política nacional de resíduos sólidos. No tocante as questões dos resíduos de serviços de saúde o quadro é ainda mais grave, visto as fortes dificuldades de políticas públicas adequadas e de orçamento para fazer valer os aspectos normativos. Diante desse quadro, o presente estudo busca realizar uma releitura de um diagnóstico realizado em instalações de serviços de saúde, realizada em 1999, cujo parecer apontava para inúmeras anomalias e sérios riscos ambientais e a saúde publica. Nesse contexto, foram aplicados questionários nos mesmos estabelecimentos visitados e comparados os cenários. Com base nos resultados obtidos foi possível avaliar que a introdução de novas legislações não evidenciou avanços nos quadros diagnosticados anteriormente.
\end{abstract}

Palavras-chave: Resíduos Sólidos. Serviços de Saúde. Gerenciamento. Destinação Final.

\begin{abstract}
The problem with solid waste is a frequent theme in major scientific research in the environmental area, however, this issue under the legal standpoint only had strong advances with the publication of CONAMA resolutions, already dated in the twenty-first century and the recent publication policy domestic solid waste. Regarding the issues of waste from health services the picture is even more serious, since the strong difficulties of adequate public policies and budget to enforce the regulatory aspects. Given this situation, this study tries to make a remake of a diagnosis made in health care facilities, in 1999, whose report pointed to numerous anomalies and serious environmental and public health risks. In this context, questionnaires were administered in the same establishments visited and compared the scenarios. Based on the results it was possible to evaluate the introduction of new laws not improved frames diagnosed earlier.
\end{abstract}

Keywords: Solid Waste. Health Services. Management. Final Destination. 


\section{INTRODUÇÃO}

A preocupação mundial com o meio ambiente cada vez, mas é um assunto que vem preocupando a população de um modo geral. A necessidade de implantar políticas de gerenciamento dos resíduos dos serviços de saúde (RSS), nos diversos tipos de estabelecimentos de saúde é imprescindível.

A falta de informações sobre o assunto, conforme relatam Naime et al (2004), é um dos principais motivos para a ausência de projetos bem sustentados que determinem melhorias no setor. Particularmente os resíduos dos serviços de saúde merecem atenção especial em suas fases de separação, acondicionamento, armazenamento, coleta, transporte, tratamento e disposição final, em decorrência dos riscos graves e imediatos que podem oferecer, particularmente na questão infecto-contagiosa.

Segundo Leite et al (2003), a ausência de definições políticas e diretrizes para a área de resíduos nos três níveis de governo (federal, estadual e municipal) associa-se à escassez de recursos técnicos e financeiros para o equacionamento do problema. Com relação às questões legais, o país passou por um processo de evolução nos últimos anos, com o surgimento de novas resoluções mais específicas para determinados tipos de resíduos considerados perigosos, especificações de padrões de emissão para processos de tratamento de resíduos como a incineração e responsabilização do gerador de alguns tipos de resíduos no gerenciamento dos mesmos.

A preocupação com o gerenciamento de resíduo em qualquer âmbito é uma realidade. Segundo Camargo et al (2009) as Instituições Hospitalares estão entre as organizações mais complexas de serem administradas. Nelas encontram-se reunidos vários serviços e situações simultâneas: hospital de serviços médicos, serviços de enfermagem, hotel, restaurante, transporte, limpeza, lavanderia, vigilância, recursos humanos e relacionamento com o usuário. Consoante isso, essas organizações são cada vez mais regidas por leis, normas, regulamentações e portarias, vindas de diversos órgãos e instituições.

Silva (2005) definiu que os resíduos de serviço de saúde, doravante RSS apesar de representarem uma pequena parcela em relação ao total de resíduos gerados em uma comunidade, são forte potenciais de propagação de doenças e apresentam um risco adicional aos trabalhadores dos serviços de saúde e a comunidade em geral, quando gerenciados de forma inadequada.

No Brasil o tema é explorado na legislação desde 1977, conforme evidenciam Brasil (1977, 1987, 1988, 1993 e 2001). Considerando a necessidade de prevenir e reduzir os riscos à saúde e ao meio ambiente, por meio do correto gerenciamento dos resíduos gerados pelos serviços de saúde, a ANVISA estabeleceu diretrizes gerais quanto ao RSS, encontrados na resolução no 23/2003. Entretanto, apenas em 2005, surge a resolução no 358/2005 do CONAMA que Dispõe sobre o tratamento e a disposição final dos resíduos dos serviços de saúde.

O Art. 3으 da Resolução no 385/2005 do CONAMA, estabelece que cabe aos geradores de resíduos de serviços de saúde e ao responsável legal, referidos no art. 10 desta Resolução, o RSS desde a geração até a disposição final, de forma a atender aos requisitos ambientais e de saúde pública e saúde ocupacional, sem prejuízo de responsabilização solidária de todos aqueles, pessoas físicas ou jurídicas que, direta ou indiretamente, causem ou possam causar degradação 
ambiental, em especial os transportadores e operadores das instalações de tratamento e disposição final. Bem como, estabelece de forma clara que os resíduos são classificados em cinco grupos: Grupo A: resíduos biológicos; Grupo B: resíduos químicos; Grupo C: resíduos radiativos; Grupo D: resíduos domiciliares; Grupo E: materiais perfurocortantes ou escarificantes. (BRASIL, 2005).

Apesar de transcorridos sete anos da imposição legal para novas condutas e direcionamentos, não são incomuns casos que relatam faltas ou falhas no cumprimento desses requisitos, especialmente em instituições públicas. Dentre alguns desses relatos pode-se citar os estudos desenvolvidos por Nazar et al (2005), Haddad (2006), Camargo (2009), Araujo et al (2012), Gouveia (2012), Ribeiro; Pisani Jr (2012) e Flores et al (2012). A maioria relata problemas no gerenciamento e nos elementos de segregação, quase sempre relacionados a falta da adoção de métodos claros de fiscalização e cumprimento eletivo das premissas legais.

Diante do arcabouço legal hoje em uso e dos diferentes diagnósticos disponíveis na literatura científica, o presente estudo visa elaborar um estudo qualitativo e comparativo de cenários. Mais especificamente, entre o relato desenvolvido por Cezar (1999) para unidades de saúde da cidade do Natal (capital do estado do Rio Grande do Norte), em situação avaliada anterior a publicação da referida resolução CONAMA 385/2005, e o caso atual observado nos mesmos locais pesquisados àquela época. Diante dos resultados obtidos espera-se avaliar a eficácia do elemento legal em questão e os principais riscos mitigados para a preservação ambiental e os elementos que circundam as preocupações de saúde pública.

\section{METODOLOGIA}

O estudo constitui-se de uma pesquisa aplicada, pois objetiva levar conhecimentos para extensão da aplicação das principais técnicas e recomendações acerca da legislação do gerenciamento de resíduos em unidades de saúde, levantadas mediante incursões investigativas.

Do ponto de vista dos objetivos, trata-se de um estudo exploratório-descritivo, o qual visa descrever a problemática em discussão, buscando caracterizar o objeto de estudo. Bem como, fazendo um estudo pretérito a cenários descritos em 2001, para o mesmo tema e nos mesmos locais estudados.

A hipótese pauta-se na possibilidade de haver alterações significativas nos casos analisados, pelo cumprimento do novo regulamento técnico e legal. Em outras palavras, espera-se que a implantação de uma legislação específica demonstre a eficácia para um correto gerenciamento dos resíduos advindos dessas atividades.

Do ponto de vista dos procedimentos técnicos, o estudo caracteriza-se como pesquisa bibliográfica, por sua elaboração partir do levantamento e análise de material já publicado, como artigos científicos, livros, relatórios técnicos, etc. e (Silva; Menezes, 2001), e como estudo de caso devido à utilização de dados de campo.

A estrutura da pesquisa consiste em:

- Formulação do problema, englobando a justificativa do estudo, a determinação dos objetivos, a contextualização da problemática e definição da metodologia; 
- Realização do levantamento teórico, que orienta a caracterização do objeto de estudo, as definições e conceitos a serem utilizados em análise e correntes de pensamentos que norteiam a hipótese da pesquisa;

- Levantamento de dados em campo, por meio de incursões investigativas nos principais estabelecimentos pesquisados por Cezar (1999);

- Estudo criterioso sobre o cumprimento dos requisitos atribuídos pela resolução CONAMA 358/2005;

- Realização de uma análise estatística dos dados obtidos pelos questionários aplicados, bem como, discussão sobre as melhorias que possam acarretar.

Os dados coletados em campos foram organizados, de acordo com a necessidade da utilização em pesquisa, e utilizados para elaboração do levantamento das principais ações de oportunidades.

Para a coleta de dados aplicou-se um questionário que buscou abranger os pontos mais importantes na caracterização do lixo hospitalar. Procurou-se trabalhar apenas com as diversas classes ou tipos de hospitais, não sendo englobado assim, as clínicas odontológicas, veterinárias, nem os postos de saúde.

Em razão da falta de recursos que tornara impossível a realização do levantamento de dados em todas as unidades hospitalares do município, escolheu-se apenas os estudados por Cezar (1999). O critério de escolha baseou-se nos seguintes aspectos:

- $\quad$ Tipo do hospital (público, privado ou filantrópico);

- $\quad$ Capacidade (baseado no número de leitos);

- $\quad$ Localização (norte, sul leste ou oeste);

- $\quad$ Situação da unidade hospitalar (novo, reformado ou antigo).

$\mathrm{Na}$ primeira etapa da pesquisa foi realizada a identificação das unidades, sendo assim, buscaram-se as seguintes informações: Tipo de hospital; Serviços prestados; Número de leitos, apartamentos, salas de cirurgias e curativos; Número total de funcionários e de funcionários destinados à limpeza. Na segunda etapa foram realizadas as quantificações e qualificações dos resíduos, conforme resolução CONAMA 385/2005. Nestes critérios, também foram avaliadas questões como: Freqüência da coleta; Equipamentos e vestuários utilizados; Tipos de embalagens usadas; Incidência de acidentes; Local e forma de acondicionamento.

Por fim, foram coletadas informações quanto ao tratamento e destinação final, em termos de: Existência de técnicas de tratamento; Meio de transporte dos resíduos; Empresa responsável pelo transporte; Local e forma de deposição final. O questionário utilizado é mostrado na Figura 1. 


\begin{tabular}{|c|c|}
\hline $\begin{array}{l}\text { 3. TIPO DE SERVIÇO: } \\
\text { ( ) Municipal ( ) Estadual } \\
\text { Privado ( ) Universitário } \\
\text { 4. RESíDUOS SóLIDOS: } \\
\text { - Há local exclusivo para armazenamento de } \\
\text { lixo? } \\
\text { ( ) SIM } \\
\text { ( ) NÃO } \\
\text { Por quê? } \\
\text { - há câmara fria para armazenamento de lixo? } \\
\text { ( ) SIM } \\
\text { ( ) NÃO } \\
\text { Por quê? } \\
\text { - Há coleta seletiva de lixo? } \\
\text { ( ) SIM } \\
\text { ( ) NÃO } \\
\text { - Existem normas de instrução escrita para } \\
\text { limpeza e manuseio do lixo produzido? } \\
\text { ( ) SIM } \\
\text { OBSERVAÇÕES: }\end{array}$ & $\begin{array}{l}\text { 5. TRANSPORTE DO LIXO: } \\
\text { - O lixo hospitalar é retirado separado do lixo } \\
\text { comum? } \\
\text { ( ) SIM } \\
\text { ( ) NÃO } \\
\text { - Existem separação do lixo hospitalar por } \\
\text { categorias? } \\
\text { ( ) SIM } \\
\text { ( ) NÃO } \\
\text { - Como é feito o transporte do lixo? } \\
\text { Recipiente com tampa/ Carrinho próprio } \\
\text { para o lixo? } \\
\text { - Há circulação exclusiva para carrinhos } \\
\text { limpos e para os sujos? } \\
\text { ( ) SIM } \\
\text { ( ) NÃO } \\
\text { - O equipamento que é usado para } \\
\text { transportar e portar o lixo são utilizados para } \\
\text { outros propósitos do hospital? } \\
\text { ( ) SIM } \\
\text { ( ) NÃO } \\
- \text { A coleta externa é feita por veículos } \\
\text { especializados? } \\
\text { ( ) SIM } \\
\text { ( ) NÃO } \\
\text { - Existe acesso exclusivo ao setor? } \\
\text { ( ) SIM } \\
\text { ( ) NÃO }\end{array}$ \\
\hline
\end{tabular}

Figura 1. Modelo do Questionário utilizado para coleta dos dados.

\section{RESULTADOS E DISCUSSÕES}

Após feitos os estudos internos do gerenciamento do RSS nos hospitais estudados, considerou-se $o$ atendimento as normas brasileiras vigentes. Foram selecionados dois hospitais: Complexo Hospitalar Monsenhor Walfredo Gurgel (hospital A), que é caracterizado como um hospital de administração pública, sendo considerado de grande porte, e a Casa de saúde São Lucas (hospital B), que tem uma administração privado, também classificado como hospital de grande porte.

$\mathrm{Na}$ avaliação foi observada que algumas etapas do gerenciamento do RSS nos dois hospitais são insatisfatórias, tanto o hospital A como o B os mesmos possuem locais específicos para o armazenamento para o lixo comum, orgânico e infectante (Figura 2), as peças anatômicas são guardadas no necrotério até o seu sepultamento, os mesmos não possuem câmara fria, pois a coleta é feita diariamente. 


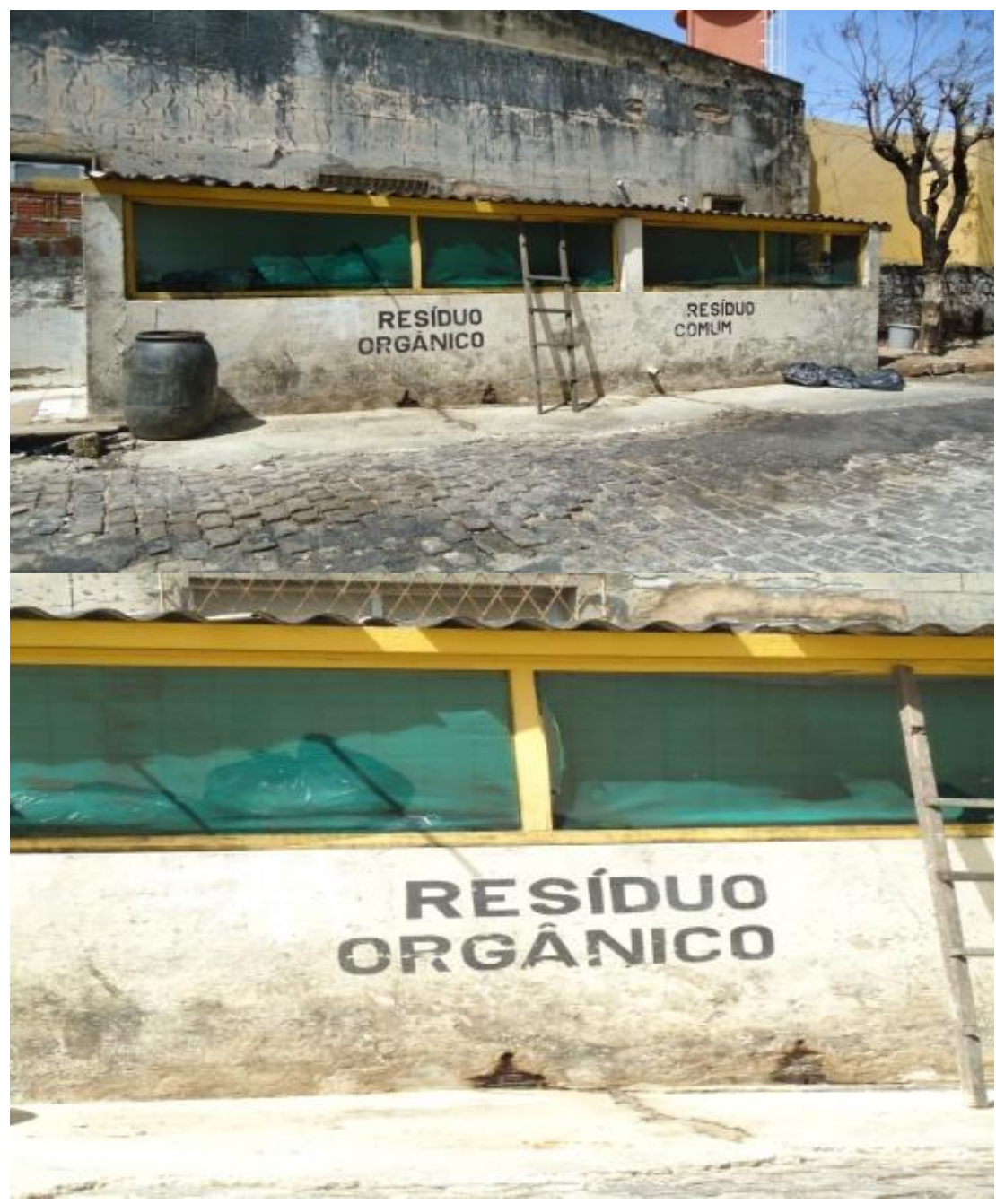

Figura 2. Resíduos Orgânicos e Comuns.

O transporte do lixo infectante até o seu local de armazenamento é feito por carrinhos específicos com tampa e rodas mesmos estando em estado precário (Figura 3), o hospital A não dispõe de local exclusivo para a limpeza de carrinhos sendo sua limpeza realizada em local impróprio sem esgoto encanado (Figura 4), o lixo é coletado no fim do dia por empresas especializadas na coleta de lixo infectante que é incinerado (Figura 5), já a coleta de lixo comum e orgânico é feita diariamente e depositado no aterro sanitário. 
2 ARANTES \& JERÔNIMO, v(11), no 11, p. 2400 - 2409, JAN-ABR 2013.

Monografias Ambientais RENOAMTSM

(e-ISSN: 2236-1308)

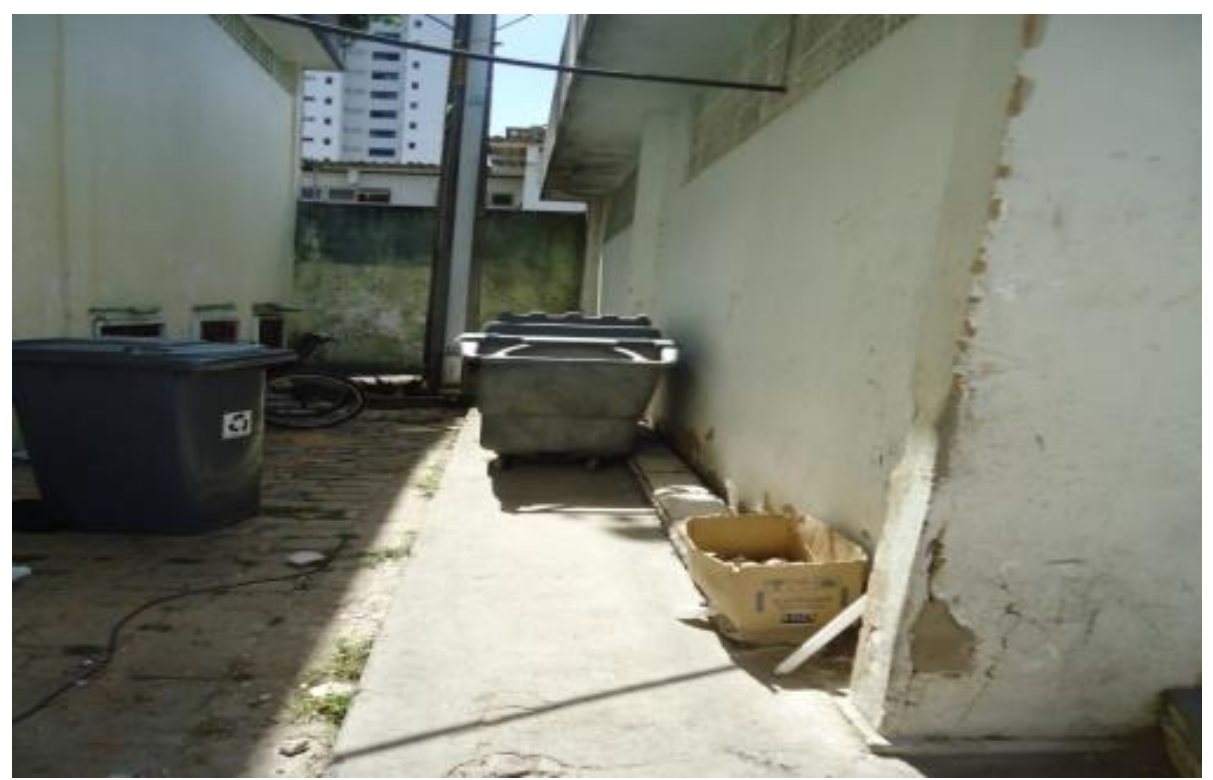

Figura 3. Contentores de resíduos.

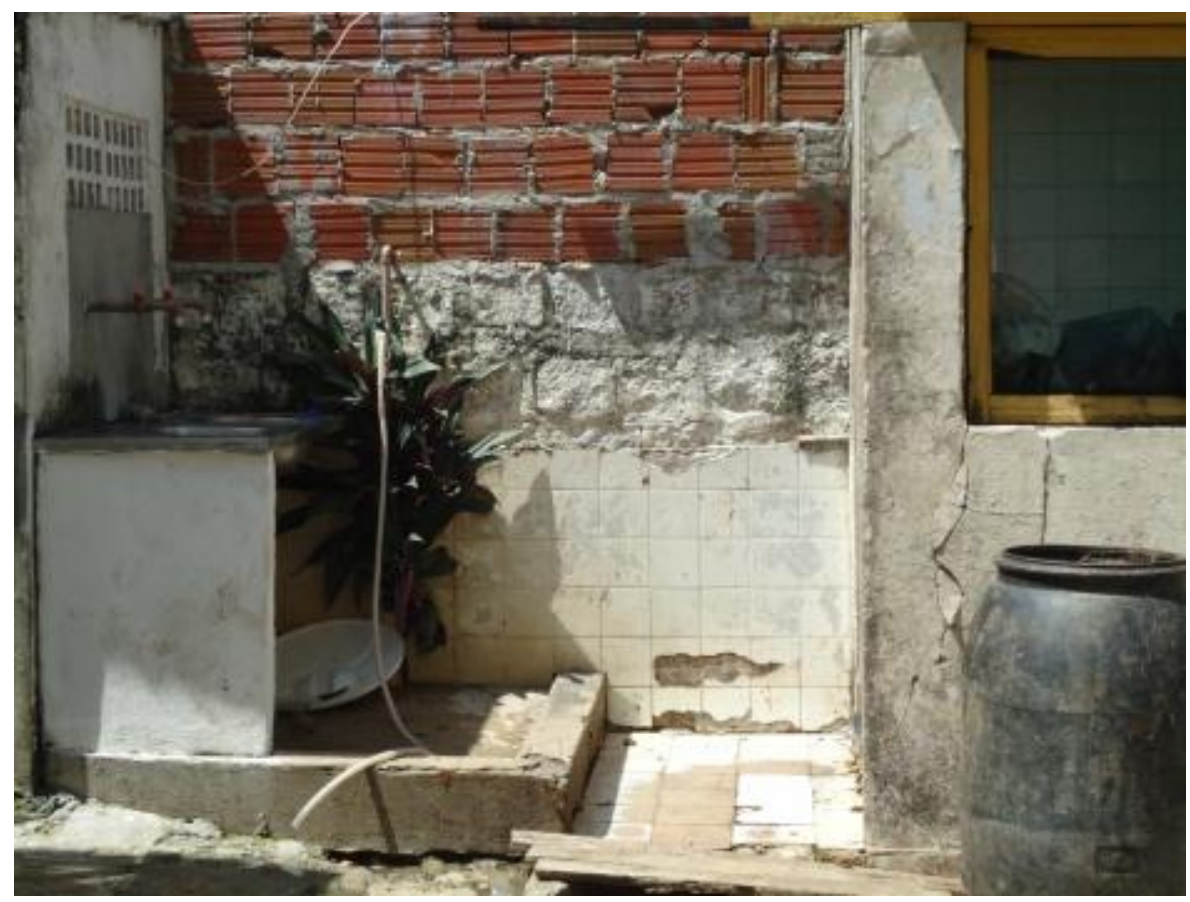

Figura 4. Área de Armazenamento. 


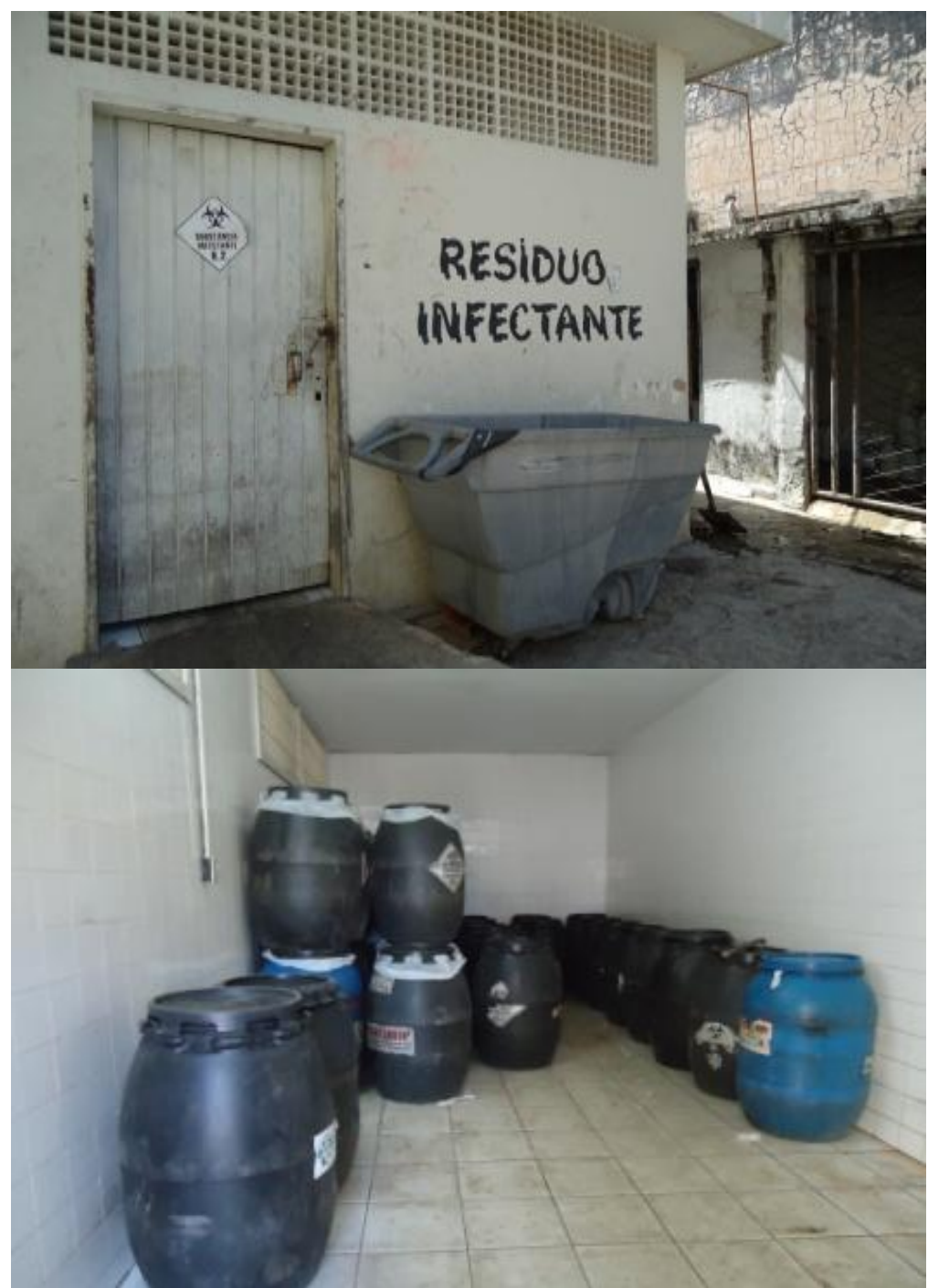

Figura 5. Resíduos Infectados.

No hospital A é produzido diariamente 1,5 t de lixo e no hospital B cerca de 600 a $800 \mathrm{~kg}$ diários, todos os andares do hospital A existem locais específicos para a coleta de todo o material que será reciclado e o material infectante, o mesmo dispõe de local adequado para o seu armazenamento e segregação, já o hospital B como não dispõe de local adequado para o armazenamento, dispõe de uma coleta seletiva diária realizada por cooperativa.

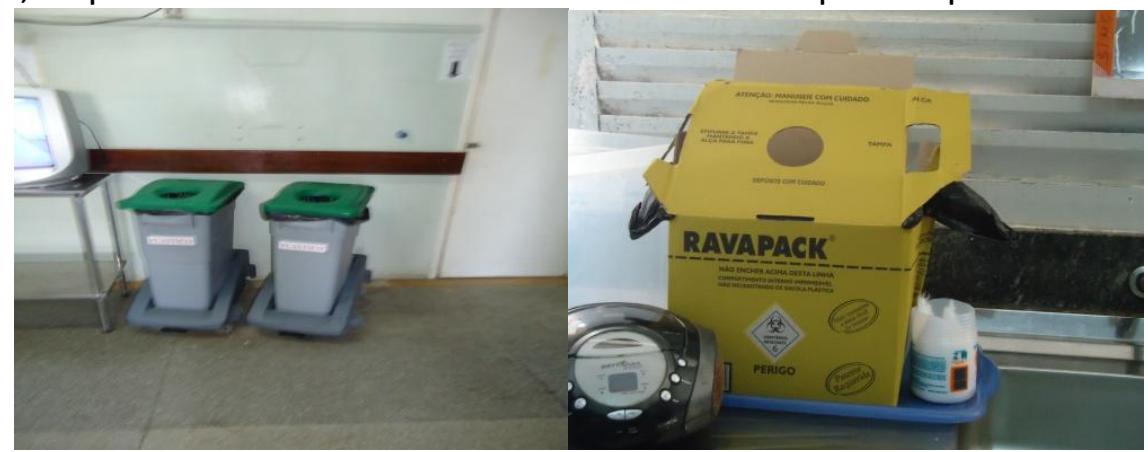

Figura 6. Resíduos Infectados.

http://cascavel.ufsm.br/revistas/ojs-2.2.2/index.php/remoa 
Os hospitais avaliados dispõem de todas as normas e instruções para a limpeza sendo feito treinamentos periódicos com os funcionários que fazem toda a coleta e segregação de todo lixo produzido, tanto no hospital A como no hospital B não possuem um local exclusivo para a circulação dos carrinhos limpos e sujos, sendo o local de circulação comum, todos os equipamentos que são utilizados para transportar e portar o lixo não são utilizados para outro propósito, bem como os funcionários que são exclusivos, o acesso é exclusiva para os funcionários. Segundo Saidelles et al (2012) entendimento entre a ciência, órgãos competentes, trabalhadores e comunidade é de fundamental importância na busca de soluções para os desafios que se apresentam frente a um ideal.

\section{CONCLUSÕES}

Os resultados demonstram que transcorridos 13 anos da avaliação realizada por Cezar (1999), e com a implantação de novas regulamentações técnicas e legais, foram visualizados avanços nos elementos de gestão e na solução de falhas absurdas no processo de adequação legal e regularização dos estabelecimentos. Os principais ganhos são observados na segregação dos resíduos e nos locais de acondicionamento temporário desses.

\section{REFERÊNCIAS BIBLIOGRÁFICAS}

ARAUJO, B. A. S. et al. Avaliação do gerenciamento de resíduos biológicos do serviço de saúde em hospitais na cidade de vitória da conquista-BA. Revista Científica, 2012.

BRASIL. Presidência da República. Ministério da Saúde. Portaria n 400 de 6 de dezembro de 1977. Aprova as normas e os padrões sobre construções e instalações de serviços de saúde, a serem observados em todo o território nacional. Normas e padrões de construções e instalações de serviços de saúde. Centro de Documentação do Ministério da Saúde, Brasília, DF, 2. ed., p. 13. 1983.

. Ministério da Saúde. Secretaria Nacional de Organização e Desenvolvimento de Serviços de Saúde. Manual de controle de infecção hospitalar. Brasília, 1987. . Constituição da República Federativa do Brasil. Brasília, DF: Senado Federal, 1988.

. Presidência da República. Conselho Nacional do Meio Ambiente (CONAMA). Resolução n. 05, de 05 de agosto de 1993. Diário Oficial da União. Brasília, 31 ago 1993. Seção I, p. 12.997.

. Presidência da República. Conselho Nacional do Meio Ambiente (CONAMA). Resolução n. 283, de 12 de julho de 2001. Diário Oficial da União. Brasília, 01 de outubro de 2001.

. Presidência da República. Conselho Nacional do Meio Ambiente (CONAMA). Resolução n. 358, de 29 de abril de 2005. Diário Oficial da União. Brasília, 04 mai 2005.

CAMARGo, M. E. et al., Resíduos Sólidos de Serviço de Saúde: Um Estudo Sobre o Gerenciamento. Scientia Plena 5, 070101, 2009.

CEZAR, G. M. Caracterização do lixo das unidades hospitalares do município de Natal/RN. IV Seminário Nacional de Resíduos Sólidos. ABES. Recife-PE. 1999.

FLORES, G. E. et al. Segregação de resíduos: o que eu tenho a ver com isso? Conferências Rede Unida, $10^{\circ}$ Congresso Internacional da Rede Unida. 2012.

GOUVEIA, N. Resíduos sólidos urbanos: impactos socioambientais e perspectiva de manejo sustentável com inclusão social. Ciência \& Saúde Coletiva, 17(6):1503-1510, 2012.

HADDAD, C. M. C. Resíduos de serviços de saúde de um hospital de médio porte do município de Araraquara: subsídios para elaboração de um plano de gerenciamento. Dissertação de mestrado. Centro Universitário de Araraquara, 2006. 
LEITE, W.C.A.; CASTRO, M.C.A.A.; SCHALCH, V. Management of solid wast in Brasil: proposal of a model that takes the management unit of water resourses as reference. IN 7TH SPECIALIST CONFERENCE CANADIAN SOCIETY ON ENVIROMMENTAL ENGENEERING - FOR CIVIL ENGINEERING. 2003. Victoria. CSCE Annul Conference. v.1. p.1-12.

NAIME, R. et al. Uma abordagem sobre a gestão de resíduos de serviços de saúde. Revista Espaço para a Saúde, Londrina, v. 5, n. 2, p. 17-27, jun. 2004.

NAZAR, M. W. et al. Gerenciamento de resíduos sólidos de odontologia em postos de saúde da rede municipal de Belo Horizonte, Brasil Ver. Panam. Salud Publica/Pan Am J Public Health 17(4), 2005.

RIBEIRO, A. B.; PISANI Jr, R. Método de obter a geração de resíduos de serviços de saúde para monitorar a execução do plano de gerenciamento de resíduos em um hospital. Revista Aidis. Vol. 5, No. 2, 2012.

SAIDELLES, A. P. F. et al. Relato de experiência sobre o gerenciamento dos resíduos de serviço de saúde. REMOA. v(8), no 8, p. 1743-1750, DOI: http://dx.doi.org/10.5902/223611706663, 2012. 